\title{
Topology-Aware Modulation and Error-Correction Coding for Cooperative Networks
}

\author{
Zhe Yang, Student Member IEEE, Lin Cai, Senior Member IEEE, Yuanqian Luo, Student Member IEEE, and \\ Jianping Pan, Senior Member IEEE
}

\begin{abstract}
User cooperation in wireless networks is inherently a cross-layer optimization problem. We identify a new direction for cooperative communications: i.e., in addition to the pointto-point communication channel between the transmitter and the receiver, the communication configuration should take the network topology into account. In this paper, we first propose a network modulation (NM) design that can transmit bits with different SNR requirements in a single symbol transmission. We then propose an error-correction coding assisted relay (EAR) scheme that is also configured according to the network topology. We study the performance of NM and EAR in both a threenode collinear network and a two-dimensional cellular network. Extensive simulations have been conducted, which demonstrate the substantial performance gain of the proposed schemes, in terms of both a higher network throughput and a lower bitenergy consumption. Comparing between NM and EAR, NM is more beneficial for the downlink performance and EAR is more beneficial for the uplink performance. Combining NM and EAR leads to a more efficient cooperative network. It is concluded that the topology-aware physical layer design will be a promising direction with many open issues for further study.
\end{abstract}

Index Terms - Topology Awareness, Network Modulation, Relay Communication, Error Correction.

\section{INTRODUCTION}

$\mathbf{F}$ OR THE WIRELESS communications among cooperative users at different locations, exploring the spatial diversity of wireless media is a promising direction but relatively under-explored. Previous approaches to address the spatial diversity can be classified into two categories. One is to take advantage of the spatial diversity gain to improve the physical (PHY)-layer performance, such as improving the spectrum and energy efficiency and reducing the bit error rate [2], [3]. The other one is to arrange multi-hop relaying and routing considering the network topology to improve the network performance, such as network throughput and end-to-end delay [4]. On the other hand, it is well known that, given the shared medium of a wireless channel, isolating communication and networking designs may lead to performance degradation. However, existing cross-layer solutions mainly focus on how to use the PHY-layer information to enhance the network protocol design, taking a bottom-up approach.

Manuscript received 15 February 2011; revised 20 July 2011. Part of the results in Section IV, related to the network modulation (NM), has been presented at IEEE Infocom 2011 [1], Shanghai, China.

Z. Yang, L. Cai and Y. Luo are with the Department of Electrical and Computer Engineering, University of Victoria, Victoria, BC, Canada (e-mail: \{zyang@ece., cai@,yqluo@ece.\}uvic.ca).

J. Pan is with the Department of Computer Science, University of Victoria, Victoria, BC, Canada (e-mail: pan@uvic.ca).

Digital Object Identifier 10.1109/JSAC.2012.120217.
We investigate the cross-layer design for user-cooperative wireless networks in a new direction. Specifically, we propose to enhance the PHY-layer modulation and error-correction coding designs considering the network topology, aimed to improve both the PHY-layer spectrum and energy efficiency, which can lead to a better network performance in terms of a higher network throughput and a lower bit-energy consumption.

The main contributions of this paper are threefold. First, we propose a network topology-aware modulation scheme, called network modulation (NM). Different from the traditional modulation schemes that are optimized for point-to-point transmissions, we design and select the NM schemes considering the network topology and the three communication channels among the source node, the destination node, and the relay node that assists the transmission. Second, we propose an error-correction coding assisted relay (EAR) scheme, where a strategically located relay can generate and transmit ReedSolomon (RS) code instead of the original information bits to the receiver. The configuration of the RS code also depends on the topology and the channels among the three nodes. Third, we further evaluate the performance of the proposed NM and EAR schemes by simulation. Simulation results show that the NM and EAR schemes can improve the network performance substantially for different topologies. For the three-node collinear topology, if the relay node is closer to the source node (i.e., the channel quality between the relay node and the source node is better than that between the relay node and the destination node), NM is more effective to enhance the performance; if the relay node is closer to the destination node, EAR contributes more to the performance gain.

The rest of the paper is organized as follows. In Sec. II, we summarize the related work. In Sec. III, we introduce the motivation and the design of network modulation, and discuss its performance and implementation. Two case studies on how to use NM for cooperative networks are also given. The errorcorrection coding assisted relay scheme is proposed in Sec. IV. The performance study of the proposed NM and EAR schemes by simulation is presented in Sec. V, followed by concluding remarks and further research issues in Sec. VI.

\section{RELATED WORK}

User-cooperative communication has been an active research topic [2], [3], [5], [6]. There are many approaches in different layers to improve the system performance. In this paper, we take a new direction to configure the modulation 
and error-correction coding schemes to enhance the network performance via user cooperation.

We propose a network modulation scheme inspired by super-positioning precoding (SPC) [7], which was designed for multicast transmissions, and recently how to use SPC for relay communications has also appeared [8], [9]. However, SPC (or h-mod) requires specialized hardware and sophisticated signal processing techniques, so it is not desirable for wireless hand-held devices. In [10], we designed a scalable modulation (s-mod) scheme that uses software mapping to rearrange the constellation of typical QAM schemes to provide differentiated services for layer-encoded video multicast over wireless networks. Here, we focus on how the softwarebased modulation remapping can be used to assist relay communications to improve the spectrum efficiency.

Using error-correction coding to improve throughput is a well-studied topic [11]-[18]. For instance, in [11], the optimal error-correcting code is selected based on time-varying wireless channel conditions. An adaptive link-level forward error correction algorithm was proposed in [12] to adjust the error-correcting codes according to the predicted packet loss rate. In [19], a partial packet recovery scheme was proposed: instead of retransmitting the corrupted packet, the sender only retransmits the bits in a packet that are likely in error and therefore the retransmitted packet length can be much smaller than that of the original packet. To overcome the shortcoming of the previous solution in [19] that requires sophisticated hardware modifications, in [20], the sender retransmits a smaller packet contains error-correcting codes. Our proposed EAR scheme is motivated by [20]. However, in a relay assisted transmission, the relay node may not have a correct copy of the original packet, which makes the configuration more complicated. In addition, the original messages and the errorcorrecting codes are transmitted through different channels. Therefore, the selection of error-correcting codes needs to consider the topology and channels among multiple nodes, and its performance gain is topology sensitive.

\section{Network Modulation for COOperative NETWORKS}

\section{A. Wireless channel model}

Wireless communication channels suffer from path loss, fading, shadowing, interference and other impairments. Denote $\kappa$ the path-loss factor at reference distance $d_{0}$. It can be calculated using the Friis free-space model: $\kappa=\frac{G_{t x} G_{r x} v^{2}}{(4 \pi)^{2} d_{0}^{2} L}$, where $v$ is the carrier signal wavelength, $L$ is the system loss factor, and $G_{t x}$ and $G_{r x}$ are the transmitter and receiver antenna gains, respectively.

Given the transmission power $P_{t}$, the received power $P_{r}$ at $d$ away can be calculated as $P_{r}=P_{t} \kappa \beta\left(\frac{d}{d_{0}}\right)^{-\alpha}$, where $\beta$ is the channel fading and shadowing factor, and $\alpha$ is the pathloss exponent, which is a constant with the value from 2 to 6 typically. $\beta$ is a random variable, depending on the fading and shadowing environment, and can be measured and estimated using training sequences. For simplicity, we assume $\beta=1$ for all links in the following calculation.

The received SNR, $\gamma$, is given by $\gamma=\frac{P_{r}}{N_{0}}=\gamma_{0}\left(\frac{d}{d_{0}}\right)^{-\alpha}$, where $N_{0}$ is the background noise power, and $\gamma_{0}$ is the received SNR at the reference distance $d_{0}$.

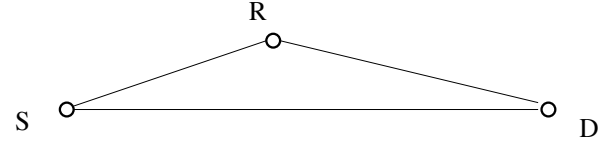

Fig. 1. A three-node topology.

\section{B. Motivation}

It is well known that relaying can improve the network performance. As shown in Section III-A, the channel quality decays quickly, proportional to $d^{\alpha}$. Thus, using $\mathrm{R}$ as a relay can result in a higher overall throughput than the direct transmission between S and D as shown in Fig. 1 .

The transmission from $\mathrm{S}$ to $\mathrm{R}$ can be overheard by $\mathrm{D}$, but because of the lower received SNR, the demodulated bits at D may suffer from a very high BER. If we design a special modulation scheme such that $\mathrm{D}$ can demodulate some of the received bits, and $\mathrm{R}$ can demodulate all the bits and further relay those bits that cannot be decoded correctly by $\mathrm{D}$ from $\mathrm{S}$ directly, the overall spectrum efficiency can be improved.

In the literature, SPC-aided relay can serve the above purpose to improve the efficiency [8]. However, as the SPC receiver is quite complicated and needs to implement iterative Successive Interference Cancellation (SIC) for demodulation, SPC has been used in the Digital Video Broadcast (DVB) standard, but not fully recommended in portable wireless systems yet. Here, we take a software-only approach to re-map the typical QAM constellation to transmit bits with different SNR requirements in a symbol. As the design and selection of the modulation consider network topology, we call the approach network modulation.

\section{NM schemes}

Considering the three-node topology shown in Fig. 1, the SNR of link SR is typically much higher than that of link SD. Therefore, the NM should maintain the similar BER for bits with different SNR. We can achieve it by selecting the constellation points of a typical QAM such that the points are clustered, and the Euclidean distances between clusters are much longer than that within the same cluster. After selecting the constellation points, we have the flexibility to assign bits in each cluster to minimize the BER of each bit.

Using this strategy, we design five NM schemes as shown in Figs. 2 and 3, based on the constellation maps of 16QAM, 64-QAM, and 256-QAM. Note that the traditional QAM modulation can be viewed as a special case of NM. Obviously, there are many other possible NM designs and we do not explore the full space of NM here, which remains a future research issue. In this paper, we study how to use these sample NM schemes and the traditional QAM schemes to facilitate user cooperation for performance improvement.

\section{BER analysis}

To obtain the BER performance of demodulated bits, we first need to identify the decision region of each symbol. The probability of a symbol is demodulated in error is equivalent to the probability that the received symbol is outside its decision 

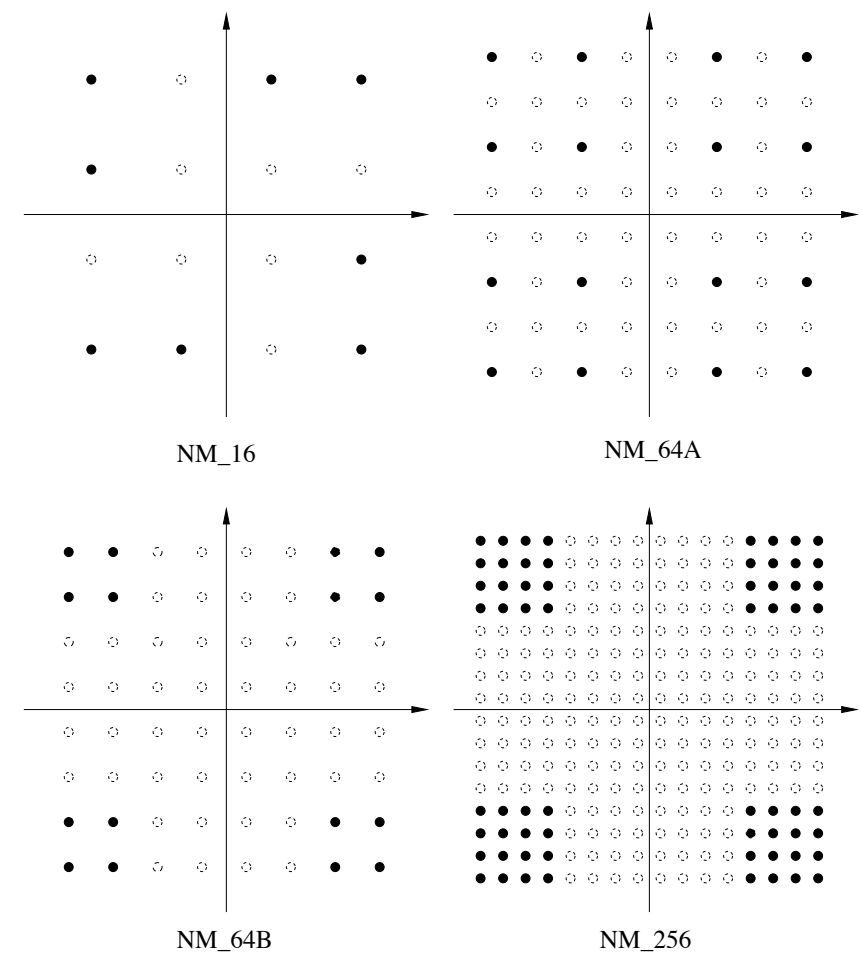

Fig. 2. Sample network modulation schemes.

region. Given the noise following Gaussian distribution, we can estimate the BER as

$$
p_{e} \approx \frac{1}{m}\left(1-\iint_{(x, y) \in R} p(x, y) d x d y\right),
$$

where $m$ is the number of bits per symbol, and $R$ is the decision region for the tagged symbol. The integral represents the probability that a received signal locates within the decision region. Therefore, if we use Gray code, the symbol error typically causes one bit error and thus the BER can be approximated by (1). The approximation comes from the assumption that there is only one bit in error for each erroneous symbol, which is acceptable if we use the Gray code for mapping bits to symbols [21].

For NM, the location of the clusters can carry $m_{1}$ layerone (L1) bits, and within each cluster, the location of the constellation points can carry $m_{2}$ layer-two (L2) bits. We can obtain the Voronoi diagram for $2^{m_{1}}$ clusters, which defines the decision boundaries of L1 symbols, and similarly the decision boundaries of L2 symbols. Then, we can use (1) to calculate the BERs for L1 and L2 bits, respectively.

\section{E. Implementation}

To implement the NM schemes, assume $\mathrm{S}$ and $\mathrm{R}$ having the knowledge of the channel conditions of SR, SD and RD. If we assume a quasi-static channel, the channel condition of $\mathrm{RD}$ estimated by $\mathrm{R}$ can be fed back to $\mathrm{S}$. At the transmitter side, we add a mapping function before modulation. Using the example in Fig. 3, $\mathrm{S}$ groups five bits and maps them to a six-bit symbol according to the standard constellation map of 64-QAM. For example, "00000" and "00111" are mapped to "000000" and "001101" in Gray-mapped 64-QAM constellation, respectively.

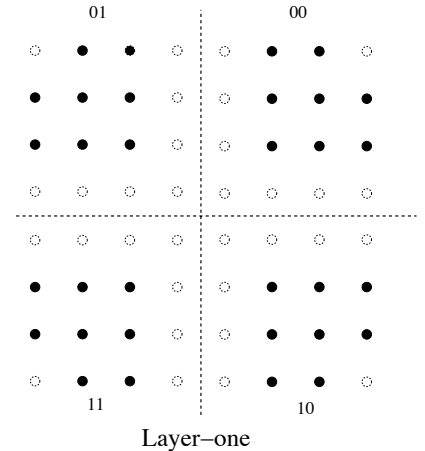

(a)

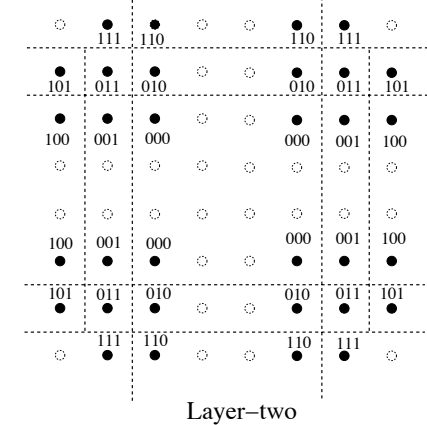

(b)
Fig. 3. Decision regions for L1 and L2 symbols for a sample NM scheme.

At the receiver side, we first define the decision regions for L1 and L2 symbols, and then demodulate each symbol to L1 and L2 bits directly. This strategy requires a minor modification to a QAM demodulator (for other options, see [10]).

\section{F. NM-assisted user cooperation}

We consider two cases that users can cooperate using NM. The first case is that $\mathrm{S}$ needs to transmit to $\mathrm{D}$ only, and a relay node $\mathrm{R}$ helps to relay the message to $\mathrm{D}$. The second one is that $\mathrm{S}$ needs to transmit to both $\mathrm{R}$ and $\mathrm{D}$, and $\mathrm{R}$ also relays for $\mathrm{D}$. The downlink transmissions in a cellular network can be an example of the second case. Next, we study how to configure NM for these two cases.

1) Case one: If $\mathrm{S}$ uses a traditional QAM to deliver $m_{S}$ bits per symbol duration (b/sym), and let $\mathrm{R}$ relay all bits to $\mathrm{D}$ with the data rate of $m_{R} \mathrm{~b} / \mathrm{sym}$, the throughput from $\mathrm{S}$ to D equals $\frac{m_{S} m_{R}}{m_{S}+m_{R}}$ b/sym. For an NM scheme, $\mathrm{S}$ can transmit $m_{S 1} \mathrm{~L} 1$ bits and $m_{S 2} \mathrm{~L} 2$ bits to $\mathrm{D}$ and $\mathrm{R}$ in one symbol duration, respectively. Then the relay node $\mathrm{R}$ transmits $m_{R}$ b/sym to relay the L2 bits to D. Thus, the throughput from $\mathrm{S}$ to $\mathrm{D}$ equals $\frac{\left(m_{S 1}+m_{S 2}\right) m_{R}}{m_{R}+m_{S 2}} \mathrm{~b} / \mathrm{sym}$.

Denote $P_{s}$ and $P_{r}$ the transmission power of source and relay node respectively, we can formulate the problem to select the best NM scheme to maximize the throughput under the BER constraint.

Problem 1: (P1)

$$
\begin{array}{cl}
\max & T h=\frac{\left(m_{S 1}{ }^{\prime}+m_{S 2}{ }^{\prime}\right) m_{R}}{m_{R}+m_{S 2}} \\
\text { s.t. } & p_{e, s d}\left(m_{S 1}, P_{s}\right) \leq p_{e} \\
& p_{e, s r}\left(m_{S 2}, P_{s}\right)+p_{e, r d}\left(m_{R}, P_{r}\right) \leq p_{e} .
\end{array}
$$

In (P1), $p_{e, \cdot}(m, P)$ is the BER given the modulation type $m$ and the transmission power $P$. As the desired $p_{e, s r}, p_{e, r d}<<$ 1 , the BER of L2 can be approximated by $p_{e, s r}+p_{e, r d}$.

As the number of modulation schemes that we can choose from is quite limited (in this paper, we consider the five proposed NM schemes and four traditional QAM modulation schemes), it is feasible to solve the above optimization problem by the search Algorithm 1, where the inputs are $p_{e}$ (the required BER), $P_{s}$ (the transmission power of $\mathrm{S}$ ) and $P_{r}$ (the transmission power of R), and the outputs are $T h$ (the throughput) and $n$ (the modulation scheme index). 


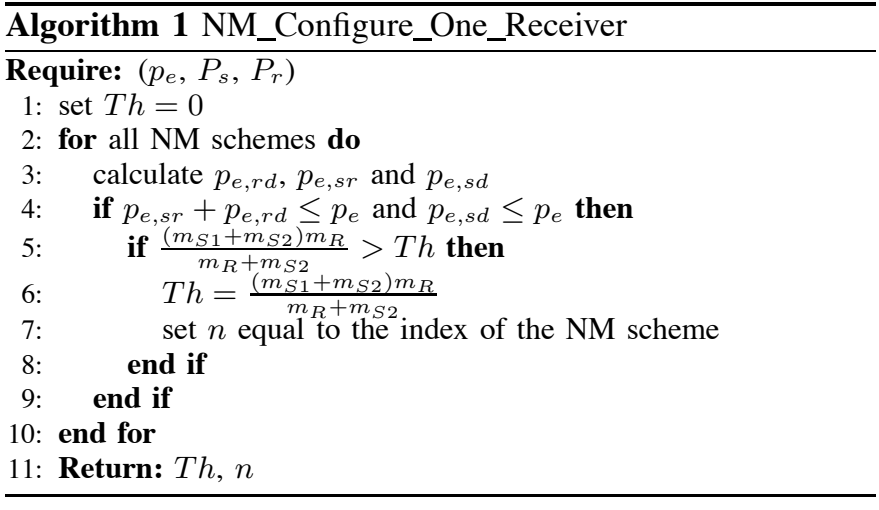

As the channel conditions of SR and RD can be different, appropriate transmission power allocation is desirable to further improve the system performance, i.e. $P_{s}+P_{r} \leq P_{\text {total }}$. Considering that the power control in a practical system is discretized with a limited number of values, we set it to be 100 in this paper and add a search loop to Algorithm 1 to find the best power allocation.

2) Case two: In this case, $S$ needs to transmit different messages to R and D, respectively. Without loss of generality, the message lengths to $\mathrm{R}$ and $\mathrm{D}$ are assumed to be the same. Using NM, S can transmit $m_{S 1}$ bits and $m_{S 2}$ bits to R and D simultaneously in one symbol duration. If $m_{S 1}>m_{S 2}$, S can transmit the remaining message to $\mathrm{R}$ using a traditional QAM modulation. If $m_{S 1}<m_{S 2}, \mathrm{~S}$ and $\mathrm{R}$ can use the NM-assisted relay in Case one to transmit the remaining message to $\mathrm{D}$. To maximize the throughput under the BER constraints, we can obtain the optimal NM configurations using Algorithm 2.

Algorithm 2 returns the throughput using the best NM schemes $(T h)$, the NM scheme selected for the two-receiver transmission $(n)$, and that for the one-receiver transmission $\left(n^{\prime}\right)$. To consider power allocation, we can add another loop to find the best power allocation strategy.

\section{ERror-Correction CODING AsSisted RElay}

\section{A. Motivation}

Still consider the three-node topology in Fig. 1. For the transmission from $\mathrm{S}$ to $\mathrm{R}, \mathrm{D}$ also receives a copy. Due to the worse channel condition of SD, the received copy by $\mathrm{D}$ may contain severe errors. In the existing solutions of relay networks, D solely relies on the transmission from $\mathrm{R}$ to decode the message from $\mathrm{S}$; in the traditional cooperative communication systems, D combines two copies of the transmissions from $\mathrm{S}$ and $\mathrm{R}$ to decode the message [22], [23]. The relay approach is simpler, but it wastes the erroneous copy from $\mathrm{S}$ to $\mathrm{D}$ entirely. Another cooperative communication approach can utilize both copies to achieve the spatial diversity gain, but it requires sophisticated signal processing and, more importantly, transmitting two copies of the same information bits increases the cost.

Here, we investigate a new direction for cooperative transmissions. The transmission takes two steps. In the first step, $\mathrm{S}$ transmits a packet to $\mathrm{R}$ and $\mathrm{D}$. In the second step, $\mathrm{R}$ uses the received packet to generate a smaller packet that contains the error-correcting code (ECC) of the original packet. Then, $\mathrm{D}$ can use the corrupted copy of the original packet from the

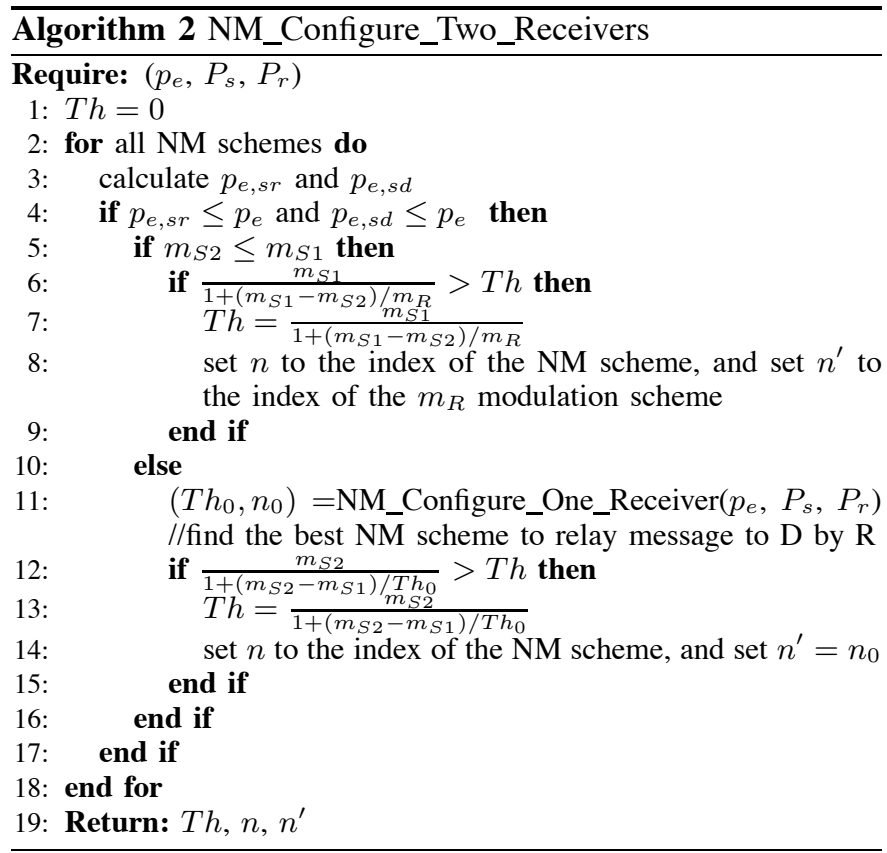

transmission by $\mathrm{S}$ and the ECC from the transmission by $\mathrm{R}$ to recover the original packet.

\section{B. Selection of ECC}

Error coding is a well developed yet still very active area. Different ECCs have different applicable scenarios considering the trade-off of overhead, performance, and implementation complexity. In traditional applications, both the message and the parity bits are transmitted over the same channel and thus with equal error probability. For EAR, the transmissions from $\mathrm{S}$ and $\mathrm{R}$ to $\mathrm{D}$ are over independent channels with different BERs due to the spatial diversity.

Given the special property of EAR, the desirable ECC should satisfy three requirements. First, it must be a systematic code, because the relay node needs to receive the original packet before it can encode it. Second, it should be scalable, so the code length can be adjusted according to different error correction requirements. Third, the computational cost should be acceptable for current hardware platforms. Considering these requirements, we choose the Reed-Solomon (RS) codes, as they satisfy the above requirements and there are many high performance software and/or hardware implementations readily available.

\section{RS-code configuration}

The RS-code is a systematic linear block code and it is organized into fixed-length blocks. Each block contains $b$ bits and the encoding and decoding operations are in Galois field $\mathrm{G}\left(2^{b}\right)$ [24]. Since modern computing systems are byte oriented, it is straightforward to choose the block size of 8 bits to be compatible with the mainstream hardware and software systems. Therefore, we use $\mathrm{GF}\left(2^{8}\right)$ consisting of $2^{8}-1$ blocks to construct the RS-code.

Denote the RS-code by a two-tuple $(n, k)$, where $n=255$ is the length of encoded blocks and $k$ is the length of message blocks (in bytes). Their difference, $r=n-k$, is the length of 
additive parity blocks appended to the $k$ message blocks. RScode $(n, k)$ can correct up to $t=r / 2$ block errors. RS-code with longer parity blocks (i.e., larger $r$ ) can correct more bit errors at the cost of a higher overhead, so we need to select the code length carefully.

Assume that RS-code is employed during all transmissions for a fair comparison. Given the BER, the block error rate, $P_{E}$, can be calculated as $P_{E}=1-(1-B E R)^{b}$. Different from the conventional configuration of RS-code in point-to-point communications, here, we need to consider the BERs of both the direct transmission and the relayed one. The corresponding block error rates are denoted as $P_{E}^{S D}$ and $P_{E}^{R D}$, respectively, which can be estimated by $\mathrm{R}$.

Given the $\mathrm{RS}(n, k)$ code, the probability that $\mathrm{D}$ can decode the $k$-block message correctly by combining the ECC from $\mathrm{R}$ and the original message from $\mathrm{S}$ is

$$
\begin{aligned}
& P_{c, E A R}(r)=\sum_{i=0}^{\frac{r}{2}}\left(\begin{array}{c}
k \\
i
\end{array}\right)\left(P_{E}^{S D}\right)^{i}\left(1-P_{E}^{S D}\right)^{k-i} . \\
& {\left[\begin{array}{l}
\frac{r}{2}-i \\
j=0
\end{array}\left(\begin{array}{c}
r \\
j
\end{array}\right)\left(P_{E}^{R D}\right)^{j}\left(1-P_{E}^{R D}\right)^{r-j}\right] . }
\end{aligned}
$$

When wireless channels are worse than the estimation and the chosen ECC is insufficient to recover the original packet, a failed transmission occurs. We have two choices: (a) to transmit another stronger ECC, or (b) to retransmit the whole packet. The first scheme may require less channel time but it is difficult to determine how many bits are in error and which ECC code is sufficient. Thus, we let the relay node retransmit the whole packet if the ECC attempt fails for simplicity.

We denote the maximum retransmission limit by $\mathrm{R}$ as $R_{\max }$, and the RS-code length for the retransmission by $\mathrm{R}$ as $r_{R D}$. The probability that the packet can be successfully received by $\mathrm{D}$ by a retransmission is

$$
P_{c, r}^{(1)}=\sum_{i=0}^{r_{R D} / 2}\left(\begin{array}{c}
k+r_{R D} \\
i
\end{array}\right)\left(P_{E}^{R D}\right)^{i}\left(1-P_{E}^{R D}\right)^{k+r_{R D}-i} .
$$

If the previous retransmission fails, the message will be retransmitted again till reaching the retransmission limit. Hence, the probability that the message can be successfully received by retransmissions is

$$
P_{c, r}=\sum_{i=0}^{R_{\max }-1}\left(1-P_{c, r}^{(1)}\right)^{i} P_{c, r}^{(1)} .
$$

Thus, for each successfully transmitted $L(r)=\left(P_{c, E A R}+(1-\right.$ $\left.\left.P_{c, E A R}\right) P_{c, r}\right) k$ bytes, the expected transmitted data length (in bytes) by $\mathrm{R}$ is

$$
\begin{aligned}
& U(r)=r+\left(1-P_{c, E A R}\right)\left(k+r_{R D}\right) \\
& \quad\left(\sum_{i=0}^{R_{\max }-1}(i+1)\left(1-P_{c, r}^{(1)}\right)^{i} P_{c, r}^{1}+R_{\max }\left(1-P_{c, r}^{(1)}\right)^{R_{\max }}\right) .
\end{aligned}
$$

Ignoring the feedback delay, given $\mathrm{R}$ using the modulation type with $m_{R D}$ bit/sym, the corresponding channel time is $T(r)=\frac{U(r)}{m_{R D}}$ symbol duration.
Based on $T(r)$, the expected throughput of the second hop (RD) is $E_{t}(r)=\frac{L(r)}{T(r)}$ (bytes per symbol duration), which should be maximized.

Discussion: As there are many efficient implementations of RS codec, the extra computation cost of EAR is moderate. Experimental results in [20] showed that the computation cost of RS encoding and decoding is less than $10 \%$ of the capacity for a commodity computer. If using FPGA to implement RS codec, the efficiency is even higher. For example, the decoding rate is up to $496 \mathrm{Mbps}$ on Spartan-II by Xilinx [25].

\section{Performance Evaluation ANd Discussion}

\section{A. Linear Topology}

We first use a linear topology, where three nodes $S, R$, and $\mathrm{D}$, are located on a line segment. The distance between node $\mathrm{S}$ and node $\mathrm{D}$ is fixed at 1 unit with the corresponding reference SNR equal to $12 \mathrm{~dB}$ (15.85). $\alpha$ is set to three, so the average received SNR at distance $x$ unit equals $15.85 \cdot(x)^{-3}$ and the AWGN channel model is considered. The tolerable BER (before error coding) is set to $p_{e}=10^{-3}$. Therefore, with direct transmission, node $\mathrm{S}$ can transmit to node $\mathrm{D}$ at most $2 \mathrm{~b}$ /sym using QPSK. The distance between node $\mathrm{R}$ and node $\mathrm{S}, x$, is varying between 0.05 and 0.95 to evaluate the system performance under different channel conditions.

1) Single-receiver case: First, we evaluate the performance of the single-receiver case, i.e., $\mathrm{S}$ transmits to $\mathrm{D}$ using $\mathrm{R}$ as a relay. In Fig. 4(a) and (b), we compare the expected throughput under the equal power allocation and optimal power allocation, respectively. In these figures, $\mathrm{x}$-axis represents the location of node $\mathrm{R}$, and $\mathrm{y}$-axis is the expected throughput (i.e., the expected number of bits received by node $\mathrm{D}$ divided by the expected number of symbol durations). For the direct transmission, the throughput is $1.984 \mathrm{~b}$ /sym considering packet retransmissions and error-control coding.

As shown in Fig. 4(a) and (b), first, to outperform the direct transmission, the location of $\mathrm{R}$ can be in a wider range using the NM-assisted relay schemes than that using the traditional relay schemes. Second, the proposed NM with a fixed transmission power can outperform the traditional relay schemes (by up to $30 \%$ for $x=0.3$ or 0.7 ); if combined with the EAR scheme, it can achieve an even higher throughput and outperform the traditional relay scheme by $50 \%$ at $x=0.7$. The improvement comes from twofold: 1) using the NM scheme, two-layered bits are transmitted simultaneously and only the L2 bits need to be relayed; and 2) with the EAR scheme, the relayed packet contains the RS parity bits, which is much smaller than the original packet.

Furthermore, compared the results in Fig. 4(a) and (b), the system throughput can be further improved by the optimal power allocation between $\mathrm{S}$ and $\mathrm{R}$, and NM/EAR can outperform the traditional relay by around $50 \%$ for $x \in(0.55,0.85)$. The results demonstrate the importance of power allocation. In the following, all the results are obtained with the optimal power allocation.

The throughput curves in Fig. 4 are in zig-zag patterns. This is because both the number of modulation schemes and the RS code length are limited and cannot be adjusted continuously. The throughput curve of the traditional relay 


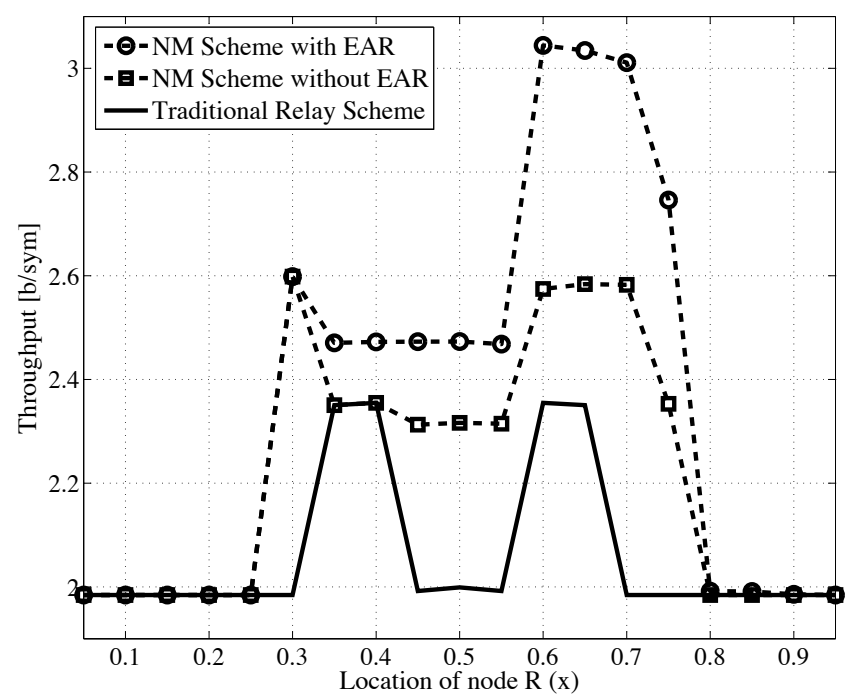

(a) Equal power allocation

Fig. 4. Throughput comparison, the single-receiver case.

scheme is symmetric with respect to $x=0.5$, while those of the NM-assisted relay schemes are asymmetric. In specific, when $x<0.5$, the performance gain is mainly from the NM; when $x>0.5$, EAR can achieve a substantial improvement. This is because, with EAR, when R is closer to D, the SNR of link SR is closer to that of SD, so we need a less strong RS code (fewer parity bits) to help D to successfully decode the packets. When $R$ is very close to $S, R$ has to retransmit all $L 2$ bits similar to the traditional relay scheme, so the performance gain of EAR largely disappears.

2) Two-receiver case: Next, let node $S$ transmit different messages to nodes $\mathrm{D}$ and $\mathrm{R}$. The throughput results are given in Fig. 5. From the figure, the NM schemes can achieve up to $72 \%$ and $91 \%$ throughut gains over the traditional schemes with or without relay, respectively. The preferable region of the relay (in which the NM-assisted relay schemes can outperform the direct transmission schemes) is wider than that for the single-receiver case. This is because that, for the two-receiver case, with NM, the data to R can be piggybacked with the transmission to $\mathrm{D}$, which can achieve a much higher throughput gain than using $\mathrm{R}$ as a relay only.

However, for the two-receiver case, the EAR scheme can only achieve a marginal gain. This is because, using NM, the percentage of the data to be relayed by $\mathrm{R}$ to $\mathrm{D}$ in the tworeceiver case is much lower than that in the previous onereceiver case.

Note that these results are sensitive to the received SNR of $\mathrm{SD}$. If the received SNR of SD changes, the desirable relay locations and the performance gains of NM and EAR also change. Nevertheless, the overall trend will remain the same.

The results with the linear topology not only provide important insights of where the performance gain comes from and for which scenarios the proposed scheme is more useful, but also indicate where the desirable relay nodes should be located.

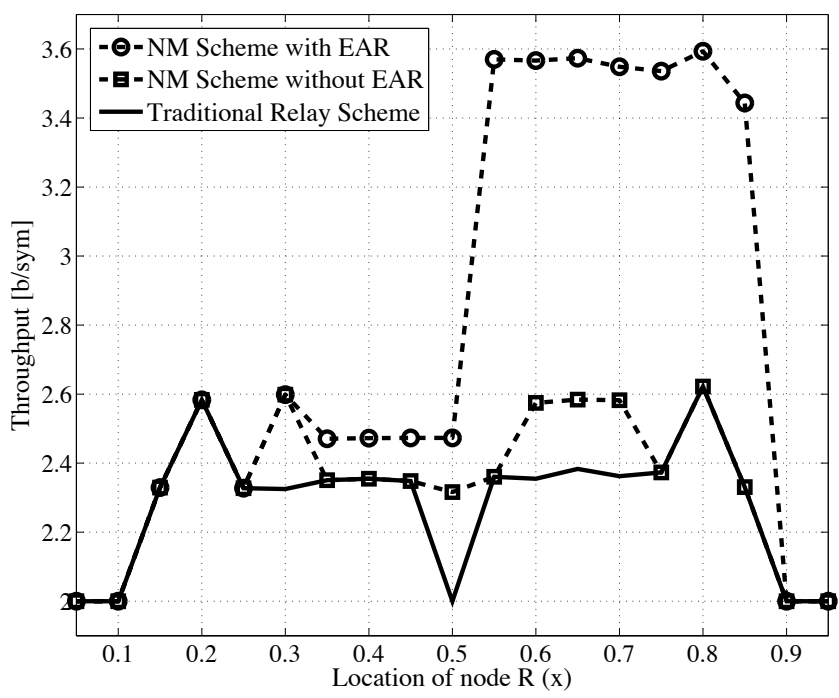

(b) Optimal power allocation

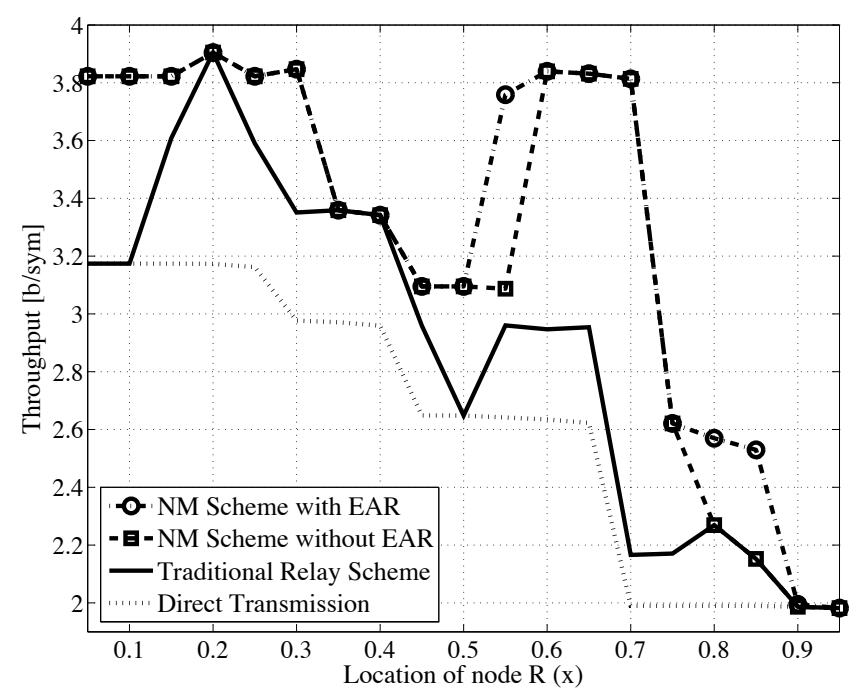

Fig. 5. Throughput comparison, the two-receiver case.

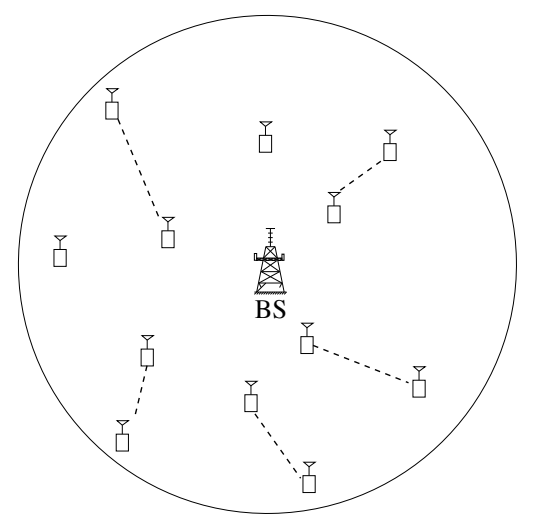

Fig. 6. Network topology. 


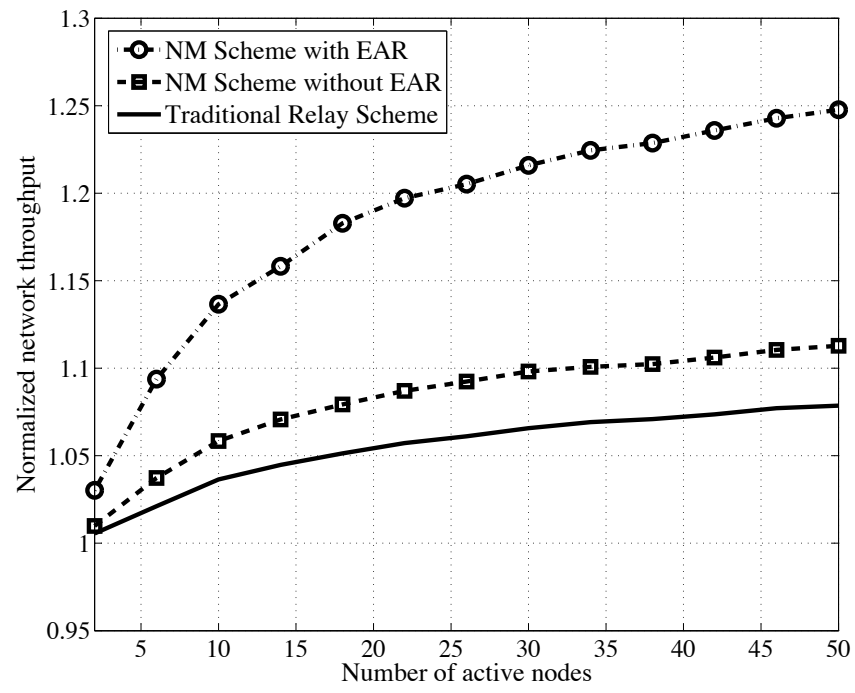

(a) uplink

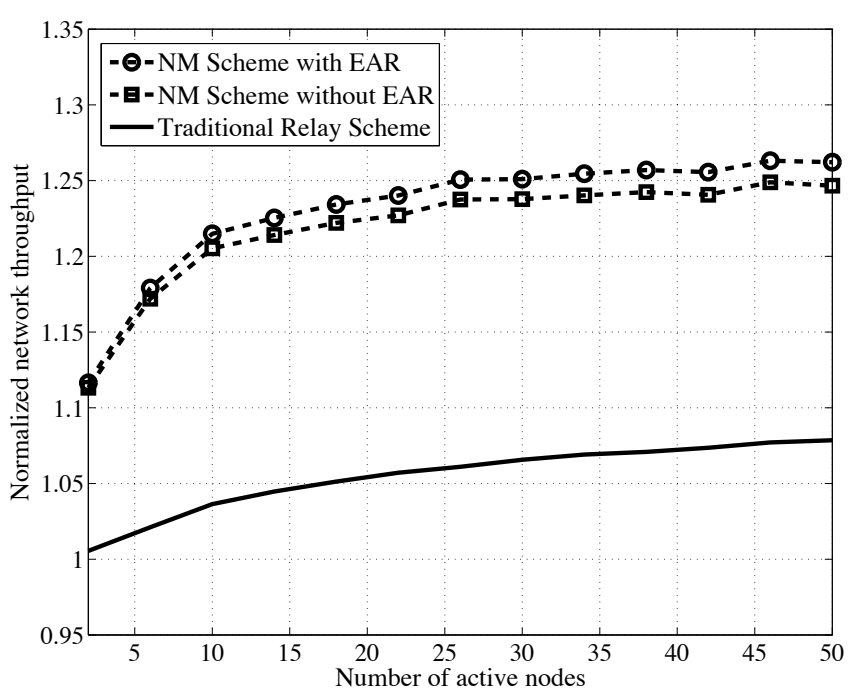

(b) downlink

Fig. 7. Network throughput.

\section{B. Network scenario}

We further evaluate the performance in a two-dimensional network, where a base station (BS) serves all the active users uniformly distributed in the cell as shown in Fig. 6. For simplicity, we assume that there is no interference for the downlink and uplink transmissions, and each node has the same amount of data to transmit for both the downlink and the uplink. Assume that the BS has the knowledge of the network topology and channel conditions that are stable during the scheduling period.

First, grouping users into pairs for cooperative transmission can be formulated as the following weighted non-bipartite matching problem, which is applicable to both the uplink and downlink scenarios. Denote the set of the mobile users in the cell by $\mathcal{F}$. Denote by $R_{i, j}$ the throughput for a pair of users $i$ and $j$, and $R_{i}$ and $R_{j}$ their (downlink or uplink) individual throughput with traditional modulations, respectively. The gain of using NM (with or without EAR) for users $i$ and $j$ is $G_{i, j}=\max \left(1 / R_{i}+1 / R_{j}-2 / R_{i, j}, 0\right)$. The optimal matching will maximize the sum of $G_{i, j}$ for all users.

If the $G_{i, j}$ for all users in $\mathcal{F}$ is known, the state-of-theart optimal matching algorithm can solve the above matching problem with the time complexity of $O\left(N^{3}\right)$ [26]. However, obtaining $G_{i, j}$ for all pairs is nontrivial. Thus, instead of using the optimal matching algorithm, we use a simple heuristic algorithm called worst-link-first (WLF) matching [27] to group users: the BS first sorts the channel qualities of all users; then it selects an unmatched user $j$ with the worst channel quality and finds another unmatched user $i$ who is most desirable for $j$ (i.e., the pair can achieve the highest performance gain); it repeats the above procedure till all users are matched. If using relay schemes (with NM and/or EAR) for a pair cannot achieve any throughput gain, the direct transmission schemes will be used. We also use the optimal power allocation for all schemes and all pairs.

1) Network throughput: Network throughput is used to evaluate the performance of different schemes, which is the

total amount of information transmitted in the whole network over the total channel time consumed.

To consider different node densities, we vary the number of active users from 2 to 50 with the step size of 4 . Set the SNR at the cell boundary as $11 \mathrm{~dB}$ (which can support QPSK using the traditional modulation scheme). For each node density, we repeated the simulation 1,000 times using different randomly generated topologies, and calculated the average results. In the following figures, the network throughputs are normalized to the one with direct transmission (which adopts the adaptive modulation scheme) for easy comparison.

The normalized network throughputs for uplink and downlink are shown in Fig. 7(a) and (b), respectively. We note that the downlink and uplink throughputs are the same with the traditional relay schemes. This is because, with the traditional relay schemes, the most preferable relay nodes are at the same or symmetric locations for the downlink and uplink transmissions. Thus, the same node will serve as the relay for both uplink and downlink transmissions. However, the throughput of downlink and uplink may not be the same with NM-assisted relay, since different relay nodes might be chosen for uplink and downlink transmissions. Thus, with NM, the workloads of relay nodes can be more balanced, which is desirable for energy-constrained wireless networks.

From the simulation results, the proposed NM with EAR scheme can achieve a $25 \%$ throughput gain when the number of nodes exceeds 50 , which is much higher than that of the traditional relay schemes.

2) Bit-energy: In addition to the throughput gain, the average bit-energy consumption is also reduced by using NM with EAR. Fig. 8(a) and (b) shows the bit-energy results, which are normalized to the bit-energy of direct transmission. With more than 30 users in the cell, for the uplink case, the NM without and with EAR schemes can reduce the bit-energy by more than $20 \%$ and $31 \%$, respectively, when compared with the direct transmission. For the downlink case, the NM scheme without and with EAR can reduce the bit-energy consumption 


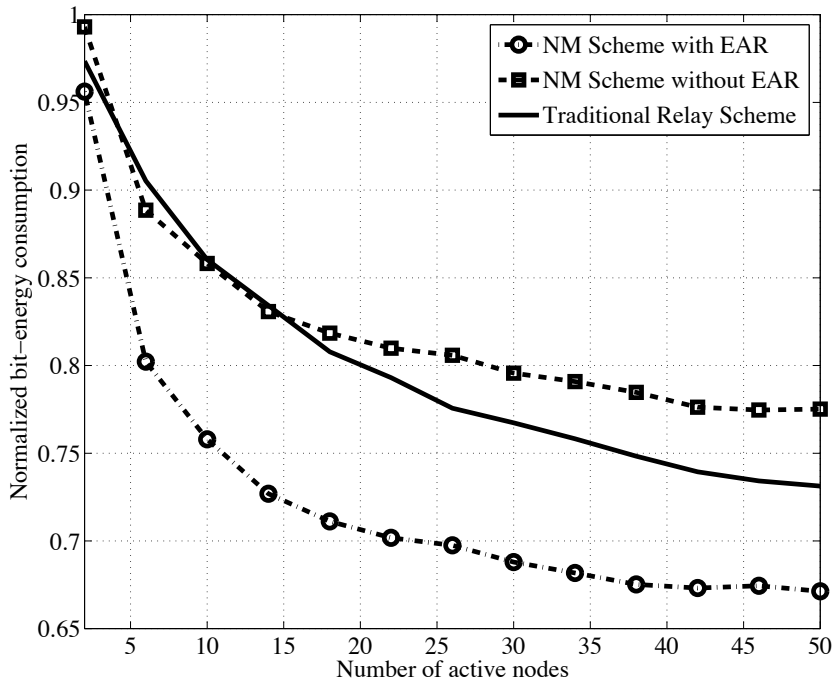

(a) uplink

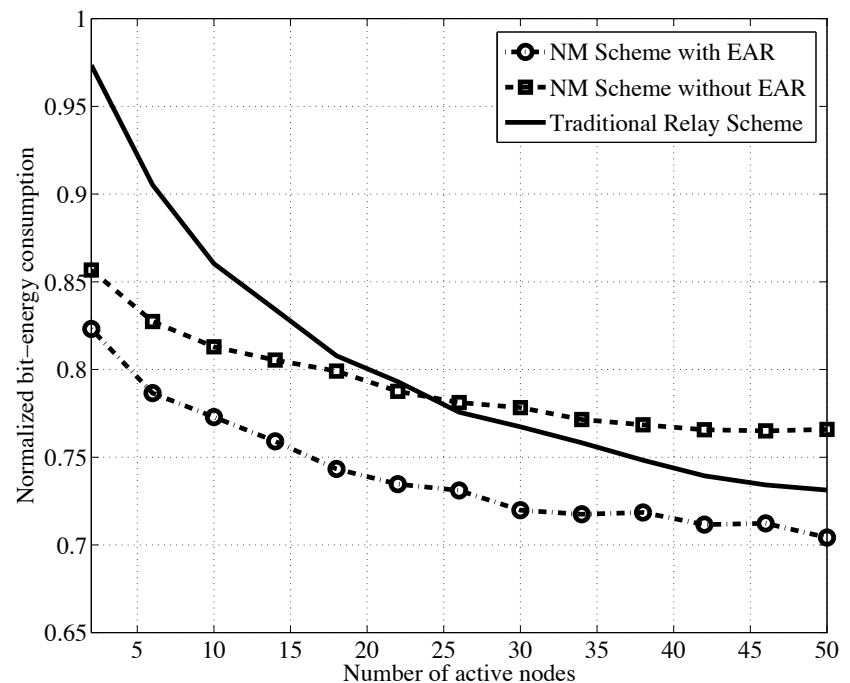

(b) downlink

Fig. 8. Bit-energy.

by more than $22 \%$ and $28 \%$, respectively. Note that there is a tradeoff between energy consumption and throughput. How to minimize bit-energy consumption while maintaining certain throughput using NM and EAR can be formulated as a different optimization problem, which can be an interesting further research issue.

3) Further discussions: The gain by the NM is more significant for the downlink transmissions while the gain by the EAR is more significant for the uplink transmissions, because the uplink scenario is similar to the one-receiver case and the downlink scenario is similar to the two-receiver case in the linear topology.

For the uplink, a higher node density can lead to a much higher gain as the performance of EAR is more sensitive to the relay location. For the downlink, even when the network only has two active users, using NM can achieve around $11 \%$ throughput gain on average.

Note that we only consider five NM schemes and the $\mathrm{RS}(255, *)$ codes in this paper. If more well-designed NM schemes and RS codes are used, it is possible to further improve the network performance.

\section{CONCLUSIONS}

In this paper, we have advocated the design and configuration of the PHY layer by considering the network topology and channel conditions among multiple nodes. We have proposed the network modulation and error-correction coding assisted relay schemes. The proposed NM is based on the mainstream QAM modem, and the proposed EAR uses the widely-used RS codes. Therefore, they are easy to be adopted in practical wireless systems. Extensive simulation results have demonstrated the substantial performance gains and lower bit-energy consumption of the proposed schemes.

It is concluded that topology-aware PHY layer design will be a promising direction with many open issues for further study. First, in this paper, we group users mainly considering the estimated channel conditions. For heterogeneous applications, the scheduling of users should consider the different utility functions for different applications. Second, the admission region of single-hop and multi-hop wireless networks with NM and EAR needs to be redefined in the new setting. Third, the interactions of NM, EAR and the protocols of other layers need further investigation. Fourth, in this work, the receiver demodulates and decodes bits from one transmission. It is possible to combine various user cooperative diversity techniques with NM/EAR to further improve the system performance by fully utilizing the spatial diversity gain. Finally, many new cross-layer optimization problems can be formulated, e.g., jointly optimize NM/EAR with relay selection/scheduling and routing, as the topology-aware PHY layer design provides one more dimension of freedom to improve the network performance.

\section{ACKNOWLEDGMENT}

The authors would like to thank the anonymous reviewers for their valuable comments that improve the quality of the paper. The authors would also like to thank Dr. T. A. Gulliver at University of Victoria, for his helpful discussions.

\section{REFERENCES}

[1] Z. Yang, Y. Luo, and L. Cai. Network modulation: A new dimension to enhance wireless network performance. In IEEE Infocom'11, 2011.

[2] J.N. Laneman, D.N.C. Tse, and G.W. Wornell. Cooperative diversity in wireless networks: Efficient protocols and outage behavior. IEEE Trans. Inf. Theory, 50(12):3062-3080, Dec. 2004.

[3] V. Mahinthan, L. Cai, J.W. Mark, and X. Shen. Maximizing cooperative diversity energy gain for wireless networks. IEEE Trans. Wireless Commun., 6(7):2530-2539, June 2007.

[4] A.E. Khandani, J. Abounadi, E. Modiano, and L. Zheng. Cooperative routing in static wireless networks. IEEE Trans. Commun., 55(11):21852192, 2007.

[5] H. Shan, W. Zhuang, and Z. Wang. Distributed cooperative MAC for multi-hop wireless networks. IEEE Commun. Mag., 47(2):126-133, 2009.

[6] W. Su, A. Sadek, and K.J. Ray Liu. Cooperative communication protocols in wireless networks: performance analysis and optimum power allocation. Wireless Personal Commun., 44(2):181-217, 2008.

[7] T. Cover. Broadcast channels. IEEE Trans. Inf. Theory, 18(1):2-14, Jan. 1972. 
[8] M. Butt, R. Zhang, S. Ng, and L. Hanzo. Superposition coding aided bi-directional relay transmission employing iteratively decoded selfconcatenated convolutional codes. In IEEE VTC'10-Spring, May 2010.

[9] P. Popovski and E. De Carvalho. Improving the rates in wireless relay systems through superposition coding. IEEE Trans. Wireless Commun., 7(12):4831-4836, 2008.

[10] L. Cai, Y. Luo, S. Xiang, and J. Pan. Scalable modulation for scalable wireless videocast. In IEEE INFOCOM-MC'10, Mar. 2010.

[11] M. Elaoud and P. Ramanathan. Adaptive use of error-correcting codes for real-time communication in wireless networks. In IEEE INFOCOM'98, volume 2, pages 548-555, 1998

[12] Y. Jin, D.G. Le, and G.W. Bai. Kalman filter-based adaptive link-layer FEC mechanism for wireless sensor networks. Application Research of Computers, 27(4):1412-1415, 2010.

[13] C.Q. Yang, E. Hossain, and V.K. Bhargava. On adaptive hybrid error control in wireless networks using Reed-Solomon codes. IEEE Trans. Wireless Commun., 4(3):835-840, 2005.

[14] T. Tran, T. Nguyen, and B. Bose. A joint network-channel coding technique for single-hop wireless networks. In IEEE NetCod'08, 2008.

[15] S. Kim, R. Fonseca, and D. Culler. Reliable transfer on wireless sensor networks. In IEEE SECON'04, pages 449-459, 2004.

[16] M.C. Vuran and I. F. Akyildiz. Cross-layer packet size optimization for wireless terrestrial, underwater, and underground sensor networks. In IEEE INFOCOM'08, pages 226-230, 2008.

[17] K. Cheun and W.E. Stark. Optimal selection of Reed-Solomon code rate and the number of frequency slots in asynchronous FHSS-MA networks. IEEE Trans. Commun., 41(2):307-311, 2002.

[18] C. Li, G. Yue, X. Wang, and M.A. Khojastepour. LDPC code design for half-duplex cooperative relay. IEEE Trans. Wireless Commun., 7(11):4558-4567, 2008.

[19] K. Jamieson and H. Balakrishnan. PPR: Partial packet recovery for wireless networks. In ACM SIGCOMM'07, Kyoto, Japan, August 2007.

[20] K.C.J. Lin, N. Kushman, and D. Katabi. ZipTx: Harnessing partial packets in 802.11 networks. In ACM MobiCom'08, pages 351-362, 2008

[21] A. Goldsmith. Wireless Communications. Cambridge University Press, 2005 .

[22] Y. Luo and L. Cai. Throughput maximization for user cooperative wireless systems with adaptive modulation. In IEEE ICC'10, 2010.

[23] S. Sharma, Y. Shi, Y.T. Hou, H.D. Sherali, and S. Kompella. Cooperative communications in multi-hop wireless networks: joint flow routing and relay node assignment. In IEEE INFOCOM'10, 2010.

[24] S. Lin and D.J. Costello. Error Control Coding. Prentice-Hall Englewood Cliffs, NJ, 1983.

[25] Antolin Agatep. Reed-Solomon solutions with spartan-II FPGAs. White Paper: Spartan-II Family, 2000

[26] H.N. Gabow. An efficient implementation of Edmonds' algorithm for maximum matching on graphs. Journal of the ACM, 23(2):221-234, April 1976.

[27] V. Mahinthan, L. Cai, J.W. Mark, and X. Shen. Partner selection based on optimal power allocation in cooperative-diversity systems. IEEE Trans. Veh.r Technol., 57(1):511-520, 2008.

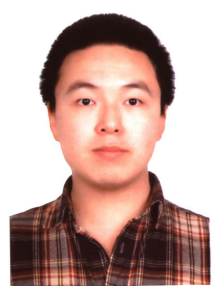

Zhe Yang (S'09) received his B.S. degree in Information Engineering in 2005 and M.S. degree in Control Theory and Engineering in 2008, both from Xi'an Jiaotong University, Xi'an, China. He is currently a Ph.D. candidate at the Department of Electrical and Computer Engineering, University of Victoria, British Columbia, Canada. His current research interests include cross-layer design for cooperative networks, scheduling and resources allocation for wireless networks and synchronization.

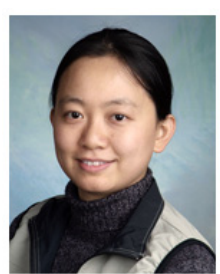

Lin Cai (S'00-M'06-SM'10) received her M.A.Sc. and $\mathrm{PhD}$ degrees (awarded Outstanding Achievement in Graduate Studies) in electrical and computer engineering from the University of Waterloo, Waterloo, Canada, in 2002 and 2005, respectively. Since 2005, she has been an Assistant Professor and then an Associate Professor with the Department of Electrical \& Computer Engineering at the University of Victoria. Her research interests span several areas in wireless communications and networking, with a focus on network protocol and architecture design supporting emerging multimedia traffic over wireless, mobile, ad hoc, and sensor networks. She has been a recipient of the NSERC Discovery Accelerator Supplement Grant in 2010, and the best paper awards of IEEE ICC 2008 and IEEE WCNC 2011. She has served as a TPC symposium co-chair for IEEE Globecom'10, and the Associate Editor for IEEE Trans. Wireless Communications, IEEE Trans. Vehicular Technology, EURASIP Journal on Wireless Communications and Networking, International Journal of Sensor Networks, and Journal of Communications and Networks (JCN).

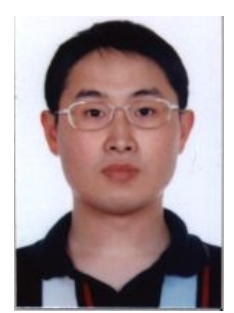

Yuanqian Luo (S'09) received his B.S. and M.S. degrees in Electrical Engineering from Southeast University, Nanjing, China, in 2005 and 2008, respectively. He is currently a Ph.D. candidate at the Department of Electrical and Computer Engineering, University of Victoria, British Columbia, Canada. His current research interests include cross-layer design and optimization for cooperative networks. $\mathrm{He}$ has received the best paper award of IEEE WCNC in 2011.

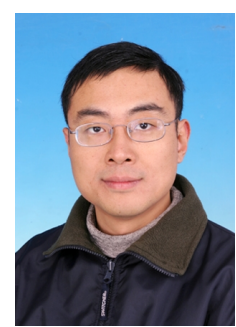

Jianping Pan (S'96-M'98-SM'08) is currently an associate professor of computer science at the University of Victoria, Victoria, British Columbia, Canada. He received his Bachelor's and $\mathrm{PhD}$ degrees in computer science from Southeast University, Nanjing, Jiangsu, China, and he did his postdoctoral research at the University of Waterloo, Waterloo, Ontario, Canada. He also worked at Fujitsu Labs and NTT Labs. His area of specialization is computer networks and distributed systems, and his current research interests include protocols for advanced networking, performance analysis of networked systems, and applied network security. He received the IEICE Best Paper Award in 2009 and the Telecommunications Advancement Foundation's Telesys Award in 2010, and has been serving on the technical program committees of major computer communications and networking conferences including IEEE INFOCOM, ICC, Globecom, WCNC and CCNC. He is a senior member of the ACM. 